Session 1: High-mass star formation near and far 


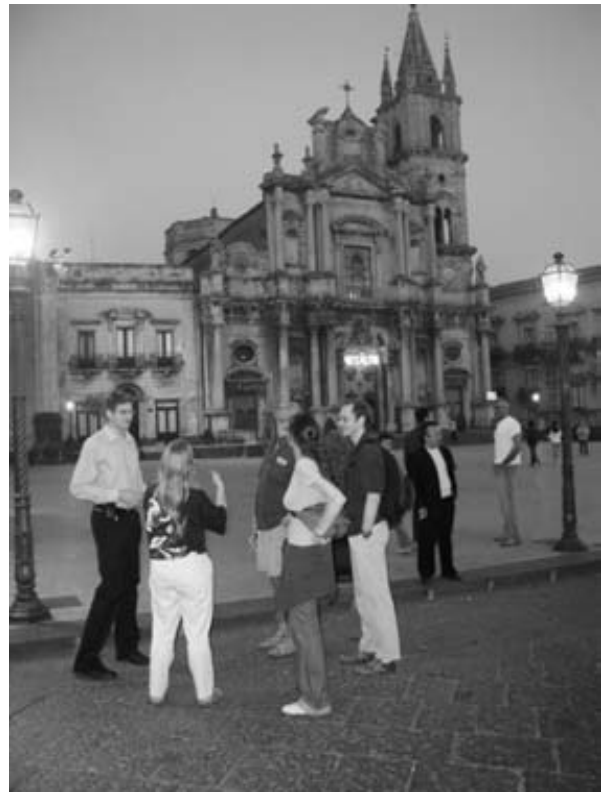

Participants touring Acireale

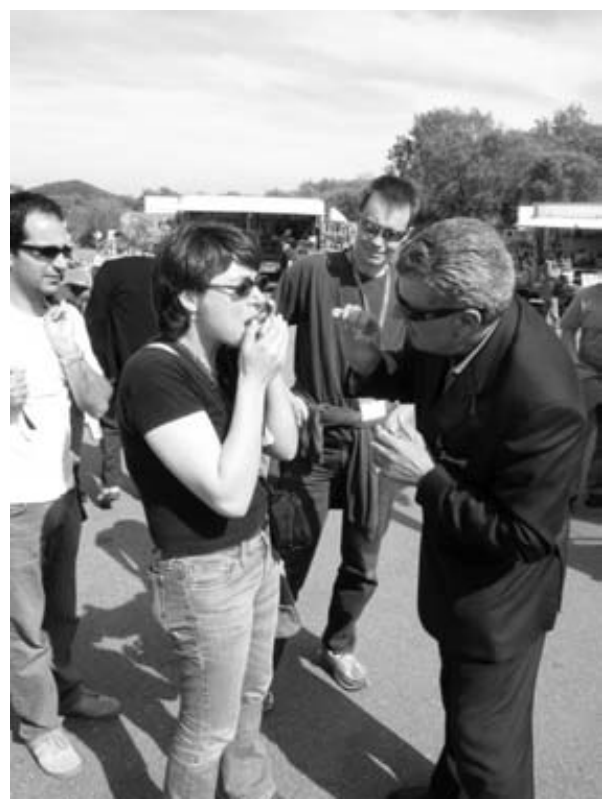

Learning how to play scacciapensieri during excursion to Etna 


\title{
The role of massive stars in astrophysics
}

\author{
Robert C. Kennicutt, Jr. ${ }^{1,2}$ \\ ${ }^{1}$ Steward Observatory, University of Arizona, Tucson, AZ 85721, USA \\ email: rkennicutt@as.arizona.edu \\ ${ }^{2}$ Institute of Astronomy, University of Cambridge, Madingley Road, Cambridge, \\ CB3 OHA, UK \\ email: robk@ast.cam.ac.uk
}

\begin{abstract}
The importance of massive stars in astrophysics is self-evident to all of us. However the full scope of this role has only become apparent over the last few years, as we begin to understand the central role that massive star formation and evolution plays in phenomena ranging from the structure and evolution of the interstellar medium, galaxy formation and evolution, nuclear activity in galaxies, and even the reionization of the universe itself. This paper briefly reviews this broad relevance of massive star formation, and the wide range in massive star formation environments found in the local universe.
\end{abstract}

Keywords. galaxies: evolution, galaxies: formation, stars: early-type, stars: formation

\section{Introduction}

This audience certainly needs no introduction to the role and importance of massive stars in astrophysics - least of all from me. Over the past few decades it has become apparent that the influence of massive stars extends well beyond the confines of their immediate stellar systems. The radiation output from massive stars ionizes their interstellar surroundings and probably have a profound influence on the subsequent formation of stars in their environments. Their mechanical energy outputs from winds and supernovae serve as energy and momentum inputs to the interstellar medium (ISM), and are now thought to sculpt the structure and energetics of the ISM in galaxies. The interplay between massive star formation and the ISM is so complex and intimate that it probably no longer makes sense in regarding star formation as the product of physical conditions in the ISM; instead the shaping of the ISM and star formation need to be regarded as parts of a larger common cyclical process. Massive stars also play a profound role as the source population for neutron stars, black holes, and the diverse set of high-energy phenomena and processes associated with those objects.

Nevertheless it has become apparent over the last 5-10 years that the reach of massive stars is even more profound than we had appreciated earlier. Observations from an armada of space facilities (Chandra, FUSE, GALEX, HST, ISO, Spitzer, WMAP, XMM) along with deeper groundbased observations from the new generation of $6-10 \mathrm{~m}$ telescopes have revealed the profound influence that concentrated injection of energy from stellar radiation, winds, and supernove can have on present-day starburst galaxies, influences that presumably replicate those that occurred in the galaxy population as a whole billions of years ago. These "feedback" processes are now thought to be important for determining the present-day mass and luminosity functions of galaxies, for determining the structure of low-mass galaxies, and for enriching galaxies as well as the intergalactic medium in heavy elements. Massive stars are even regarded now as the most likely source for reionizing the universe at redshifts of 10 and beyond, and this realization has given birth to a major industry of modeling Population III star formation. 
In this paper I shall highlight a few examples of these recent observations and theoretical results, to illustrate the broad reach and importance of the massive star formation processes that will discussed during the course of this symposium. I will also review what is known about the range of star formation environments found outside the Galaxy, to provide additional perspective on the papers that will follow. I have chosen to address the problem from an extragalactic, in part because it is the area I know best, and also because the Galactic perspective will be reviewed by the following speakers in this session.

\section{The Impact of Massive Stars on Galaxy Formation and Evolution}

It has long been appreciated that massive stars play a central role in shaping the structure and energetics of the ISM. Now thanks to the multiwavelength observational capability afforded by a new generation of X-ray, ultraviolet, and infrared satellites, combined with groundbased radio and visible observations, we are able to study these interactions directly through most of the relevant ISM phases. In actively star forming galaxies the stars can produce a cosmic "bubble bath" of interstellar holes and shells, as illustrated in Figure 1, which shows an HI map of NGC 6946 from the THINGS (The HI Nearby Galaxies Survey) survey (Walter et al. 2005, in preparation).

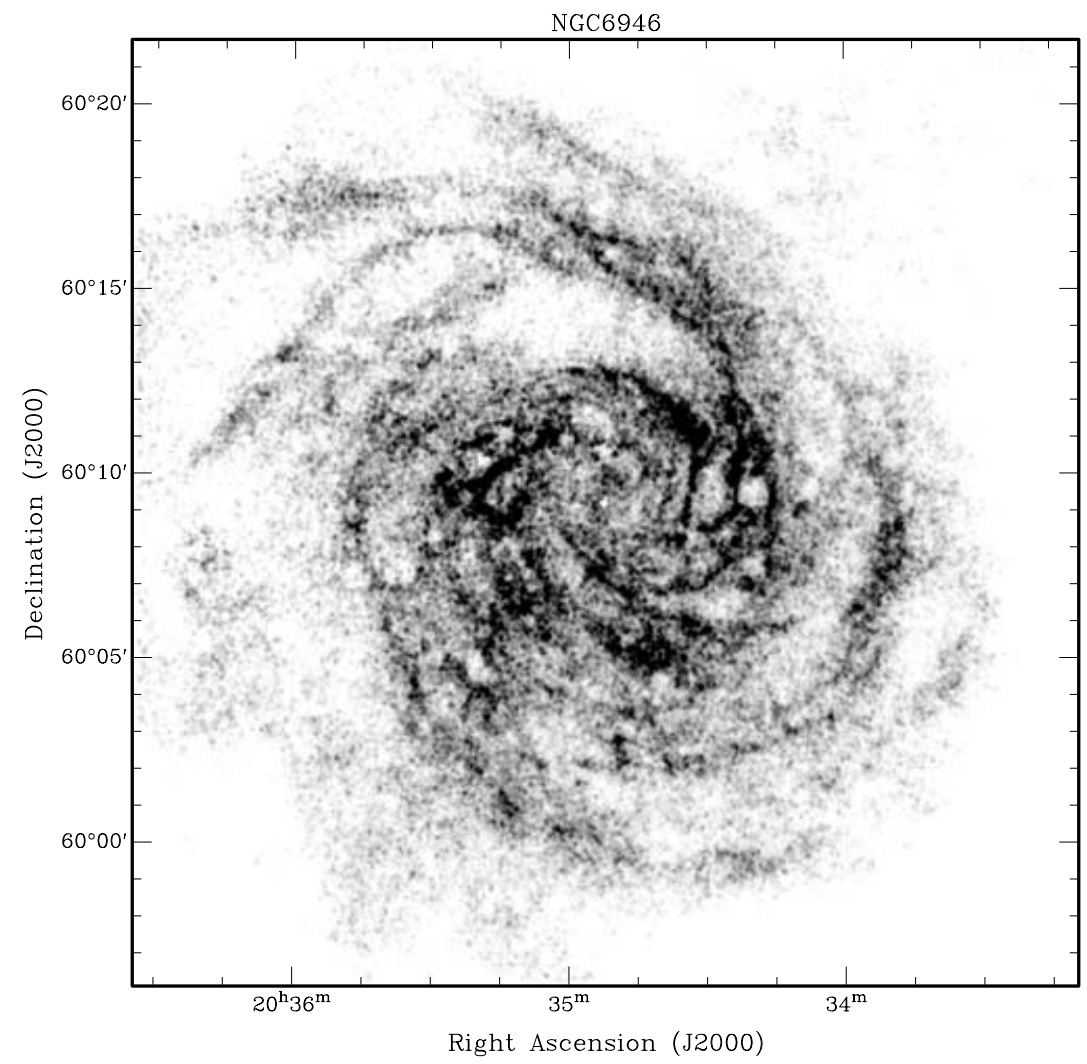

Figure 1. An HI map of the nearby Sc galaxy NGC 6946, as imaged with the VLA using the $\mathrm{B}, \mathrm{C}$, and D arrays, as part of the THINGS survey.

In most normal star-forming galaxies the effects of this feedback appear to be largely confined to the disks, but in a sufficiently massive star formation event the interstellar 
bubbles can break through into the galactic halo, and in even larger starbursts the metalenriched material in the bubble may escape the galaxy altogether. The existence of such galactic "superwinds" has been postulated for some time, but now such winds are directly observed with Chandra, XMM, and FUSE. Figure 2 shows an example in NGC 1569, a dwarf galaxy $\left(M_{B}=-17\right)$ undergoing a massive starburst. Chandra imaging and spectroscopy shows not only that the wind is being ejected from the galaxy at beyond escape velocity, but also that the material in the wind is heavily enriched in heavy elements (Martin et al. 2002). Independent evidence for such winds in other galaxies has been found from OVI absorption measured with the FUSE satellite (e.g., Heckman et al. 2001).

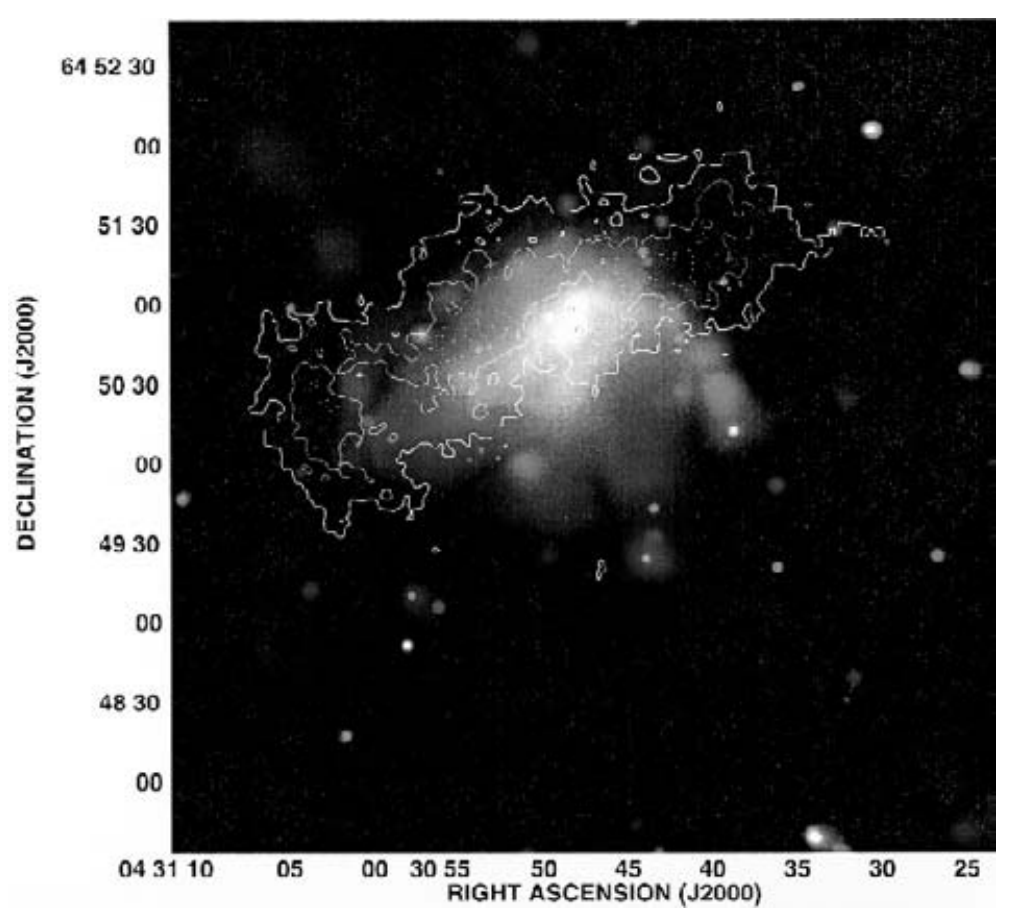

Figure 2. X-ray wind from the starburst dwarf galaxy NGC 1569, superimposed on HI contours. Adapted from Martin et al. (2002).

The starburst strength required to produce such large-scale escape of gas and metals is strongly dependent on the mass and potential well depth of the parent galaxy (e.g., Martin 1999). At the present cosmological epoch dwarf galaxies are the only objects with shallow enough potential wells to be able to sustain spontaneous mass loss from self-generated starbursts. However much more massive outflows are observed in mergerdriven starbursts, such as those found in infared-luminous and ultraluminous starbursts (e.g., Rupke et al. 2002, Martin 2005). Mass loss from such starbursts can easily exceed the mass consumed in star formation itself. These extreme events probably are excellent analogs to the primeval winds that occurred in the formation of massive spheroidal galaxies, and appear to be seen in many submillimeter-selected high-redshift galaxies (e.g., Swinbank et al. 2004). 
The impact of such primeval winds on the galaxy population as a whole may have been profound. A longstanding problem in galaxy formation is the departure of the mass and luminosity distributions of present-day galaxies from the mass spectrum of dark matter halos in the canonical CDM paradigm. This is illustrated in Figure 3, taken from Somerville \& Primack (1999). The CDM models predict far many more low-mass halos than are observed. The favored explanation now is feedback from massive star formation in dwarf galaxies, which preferentially disperse the lowest-mass systems. Massive starbursts may also have a role in explaining the relative absence of very high-mass galaxies as well, but currently the favored explanation for that deficit is nuclear activity (e.g., Di Matteo et al. 2005).

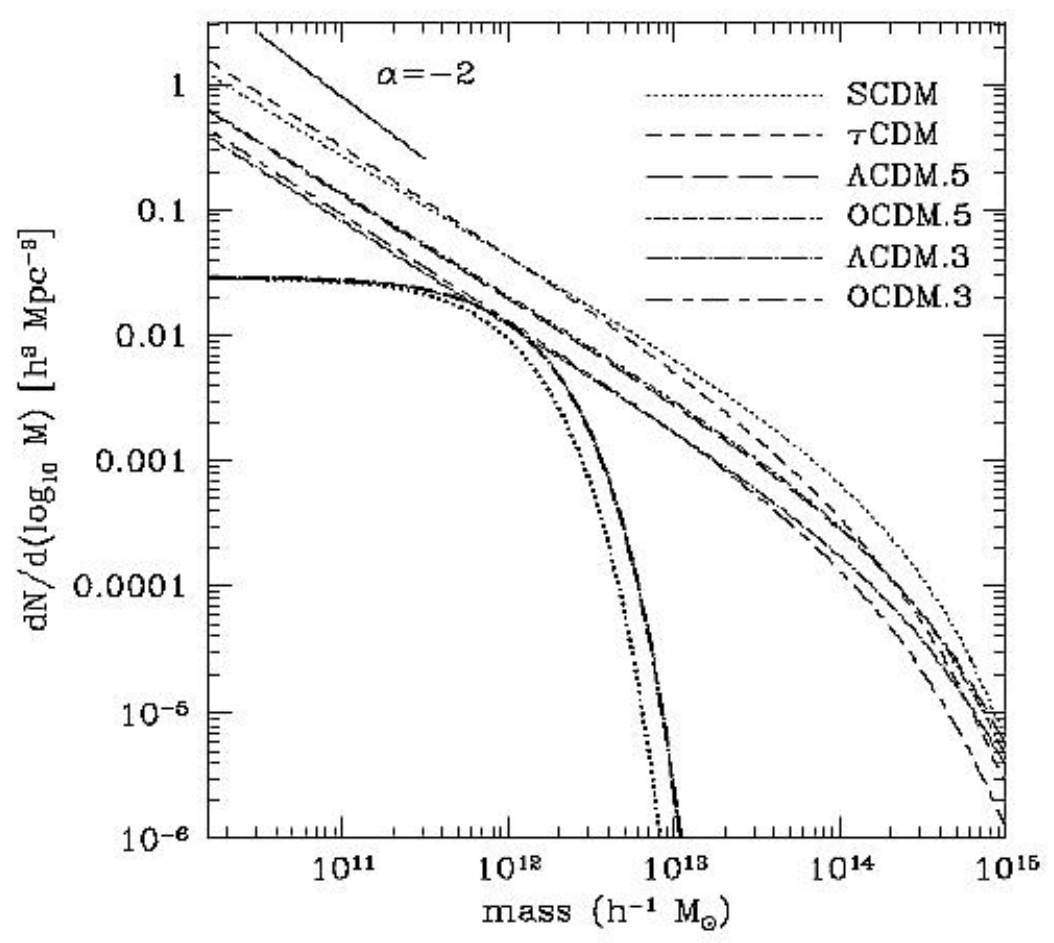

Figure 3. Comparison of the observed mass function of galaxies (lower curves), with predictions of the dark matter halo mass distribution for different cosmologies, from Somerville \& Primack (1999). The relative deficit of low-mass galaxies is now thought to arise from feedback from massive star formation, which expells gas and disperses low-mass systems.

In present-day galaxies the most massive starbursts occur in the central kiloparsecs of galaxies, usually as the result of gas mass transfer from a bar or a tidal interaction/merger (e.g., Kennicutt 1998a, Kormendy \& Kennicutt 2004). Until recently it has been difficult to separate the circumnuclear star formation from associated AGN activity, due to the combined handicaps of limited spatial resolution and dust extinction in these compact, dense regions. However enormous progress has been made over the past ten years due to the ability of the ISO and Spitzer telescopes to penetrate the dust and obtain spectral diagnostics of the circumnuclear gas, and the ability of HST to detect the spatial signatures of circumnuclear star formation in less-obscured systems. Nuclear dust spirals are a 
nearly ubiquitous feature in disk galaxies with (and without) active nuclei, and circumnuclear star formation is detected within a few hundred parsecs or less of a large fraction of active nuclei (e.g., Martini et al. 2003), though evolved stars still dominate the nuclear populations in most objects. Infrared spectroscopy with ISO and Spitzer likewise reveal that most ultraluminous infrared "starburst" galaxies actually appear to be powered by a combination of circumnuclear starbursts and a bright accretion-powered active nucleus (e.g., Genzel et al. 1998).

Finally, massive star formation has become one of the hottest subfields of cosmology, with the realization that radiation from massive stars is the most likely explanation for the reionization of the universe at redshifts $z=6-20$. Star formation at such epochs would have taken place under conditions radically different from those today, with nearzero metal abundances, little or no magnetic fields, and in a cosmological radiation bath at least 10 times higher than the present $2.7 \mathrm{~K}$ background. An example of a simulation of the formation of the "first stars" is illustrated in Figure 4 (for a related paper see Abel, Bryan, \& Norman 2002).

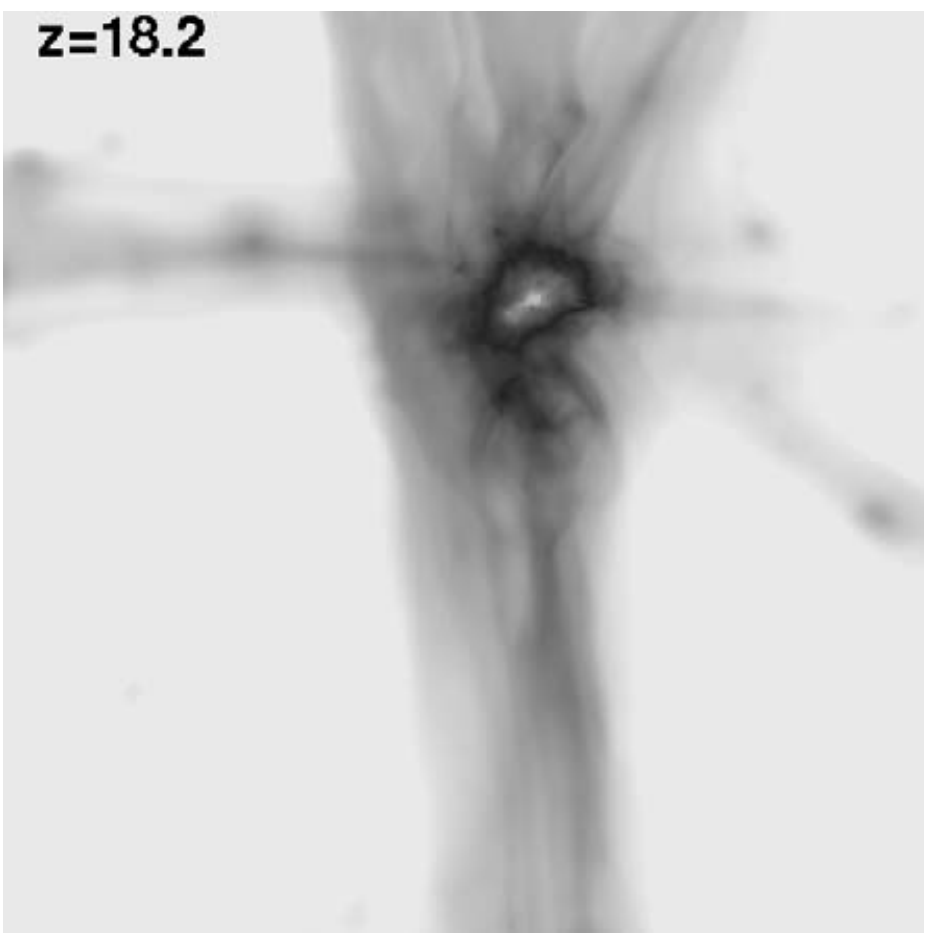

Figure 4. Simulation of formation of a zero-metallicity pre-galactic object at redshift $z=18$ by Abel, Bryan, and Norman.

\section{The Diversity of Massive Star Forming Environments}

One of the recurring themes in the preceeding discussion is the vast range of physical conditions and interstellar environments in which massive star formation is expected to play a major role. In order to provide some perspective for the papers that will follow I will finish my review with a discussion of the range of star formation rates and star formation region properties found in the local universe and at earlier epochs.

The Milky Way galaxy appears to have a typical star formation rate (SFR) for an intermediate-type spiral galaxy $\left(\sim 3 \mathrm{M}_{\odot} \mathrm{yr}^{-1}\right.$, e.g., McKee \& Williams 1997), and it offers 


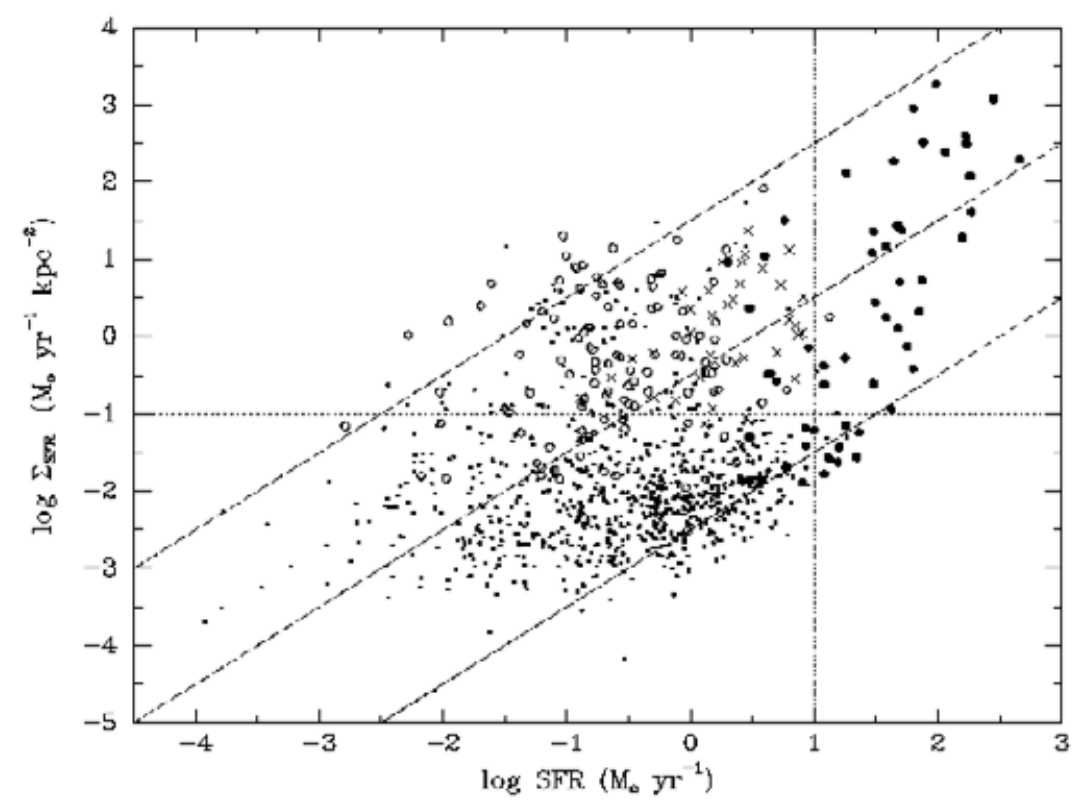

Figure 5. Distribution of SFRs and SFR densities in local star-forming galaxies. Small points are normal galaxies taken from the Arizona 11HUGS survey, James et al. (2004), Hameed \& Devereux (1999), and the GoldMine database, as described in the text. Overplotted are three types of starburst galaxies, luminous and ultraluminous star-forming infrared galaxies (large solid points), blue compact starburst galaxies (open circles), and circumnuclear starbursts identified in the optical (crosses). The diagonal lines denote constant radius of the star-forming regions, from 0.1 to $10 \mathrm{kpc}$ (top to bottom). The horizontal and vertical dashed lines denote the typical values delineating starburst galaxies, as discussed in the text.

a wide spectrum of massive star formation environments, ranging from small associations containing a single OB star to complexes with of order 100 O-stars, such as NGC 3603 and the Arches cluster (e.g., Figer et al. 2002, Stolte et al. 2004). Similarly the Galactic regions span a wide range in local densities and obscurations, ranging from very young and deeply embedded ultracompact HII regions to evolved clusters that are in the process of dispersing their parent molecular clouds.

The present-day galaxy population has a broad range of star formation properties, and a more diverse population of individual star-forming regions. The diversity in global star formation properties is illustrated in Figure 5, from Kennicutt et al. (2005, in preparation). Plotted on the $\mathrm{x}$-axis are total SFRs, which range from of order $10^{-4} \mathrm{M}_{\odot} \mathrm{yr}^{-1}$ in dwarfs and gas-poor early-type galaxies to $10 \mathrm{M}_{\odot} \mathrm{yr}^{-1}$ in normal galaxies (small points), and up to $1000 \mathrm{M}_{\odot} \mathrm{yr}^{-1}$ in ultraluminous infrared galaxies (large solid points). The y-axis parametrizes the star formation in terms of the average surface density, defined simply as the ratio of the SFR to the deprojected area of the disk containing over $95 \%$ of the star formation. This too ranges over orders of magnitude, with normal disk galaxies having a relatively narrow range around $0.001-0.1 \mathrm{M}_{\odot} \mathrm{yr}^{-1} \mathrm{kpc}^{-2}$ and starburst galaxies extending this range by another 4 orders of magnitude.

As might be expected there is a similar diversity in the physical properties of the star forming regions as well, and with the arrival of large datasets in the UV and infared 
from GALEX and ISO/Spitzer, respectively, we are now able to probe the systematic properties of these populations. The mass spectrum of young associations and clusters extends up to ionizing luminosities in excess of $10^{53} \mathrm{ph} \mathrm{s}^{-1}$, corresponding to estimated cluster masses of $10^{6} \mathrm{M}_{\odot}$; direct evidence for such "super star clusters" are observed in many of the most extreme starbursts. These may well be the formation environments of long-lived globular clusters in many galaxies, though the efficiency of such cluster formation must be very low (Kennicutt et al. 2002). These luminous clusters are also observed at all evolutionary stages, ranging from highly obscured objects identified only in the radio continuum or infrared (e.g., Kobulnicky \& Johnson 1999) to supergiant HII regions.

The compact HII regions represent an especially interesting class of objects. The embedded clusters often exceed the 30 Doradus nebula in luminosity, with of order $10^{3}$ O-stars. The surrounding gas clouds have densities of several thousand $\mathrm{cm}^{-3}$, yielding Strömgren radii of a few parsecs, and high optical depths even in the thermal radio continuum. Interestingly they are not limited to metal-rich environments. Indeed one of the most famous examples, in the dwarf galaxy SBS 0335-052, resides in the second most metal-poor galaxy known (see the poster by R. Thompson et al. in this volume). At the other extreme are a class of UV-bright star forming regions identified recently with the GALEX satellite, which reside in the outer disks of galaxies and appear to have no surrounding nebulosity whatsoever (e.g., Thilker et al. 2005).

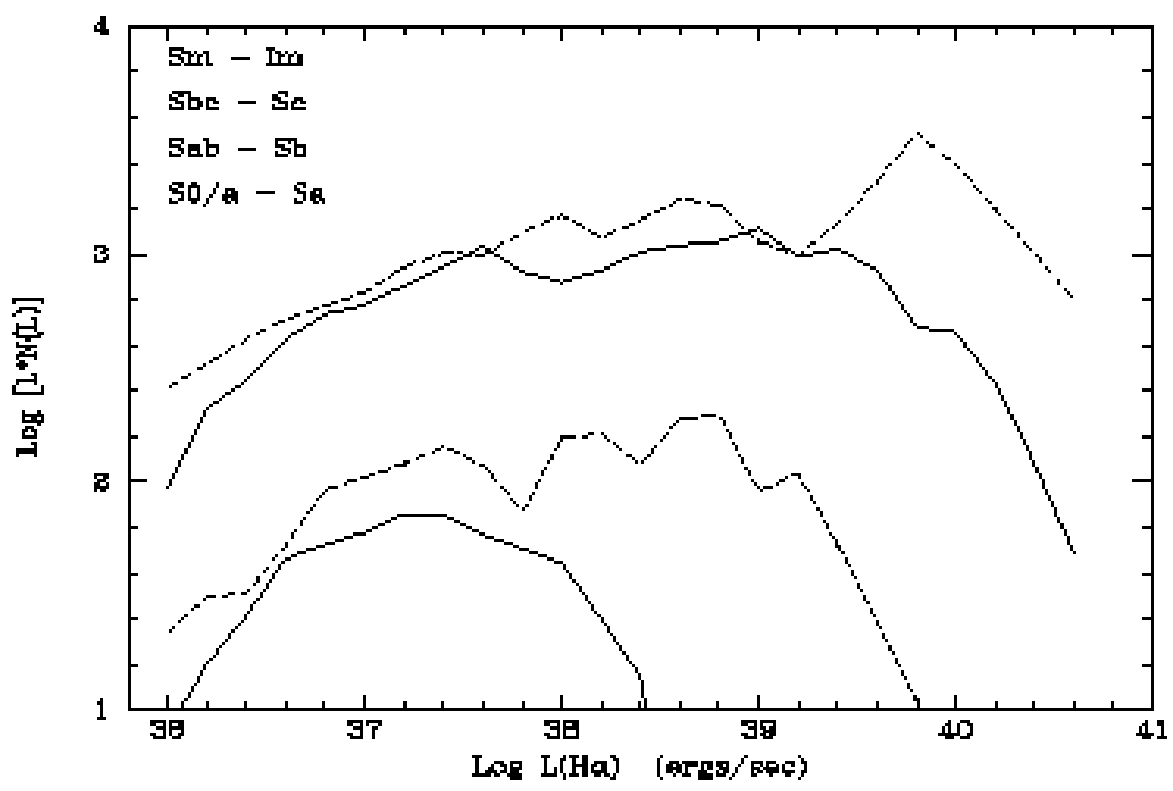

Figure 6. Composite luminosity-weighted differential HII region luminosity functions, plotted as a function of RSA galaxy type (labels show order of curves). For reference the Orion nebula cluster produces an extinction-corrected luminosity of $\sim 10^{37} \mathrm{ergs} \mathrm{s}^{-1}$, while corresponding value for the 30 Dor cluster is $\sim 10^{40} \mathrm{ergs} \mathrm{s}^{-1}$. 
Thanks to the collection of systematic multi-wavelength datasets with the Spitzer Infrared Nearby Galaxies Survey (SINGS) (Kennicutt et al. 2003) and other surveys some trends in the properties of the massive star forming regions are beginning to emerge. One important trend already seen 15 years ago is a shift in the mass distribution of starforming regions to more massive objects in later-type galaxies with higher SFRs (e.g., Kennicutt et al. 1989, Bresolin \& Kennicutt 1997, Larsen 2002). This is illustrated in Figure 6, which plots the luminosity-weighted distribution of HII region luminosities in galaxies ranging from type Sa to Irr. This change is very significant because it means that most star formation in early-type disks occurs in small associations where massive stars influence the ISM in isolation, whereas in late-type disks the average massive star forms in a cluster or assocation with several dozen or hundred others, and there is a much higher potential for collective feedback effects on the ISM. The underlying physical parameters driving these changes are not well understood, but there are suggestions that the mass spectra of the star forming regions are most strongly correlated with the specific SFR itself (Larsen 2002).

Analysis of SINGS data also reveals pronounced differences in the frequency of highdensity embedded star forming regions between galaxies. Pronounced differences have been observed for some time between the population of star forming regions in disks and the high-density circumnuclear environments (e.g., Kennicutt 1998a). But we now see differences within disks of different galaxies that bridge these extremes. For example in most intermediate to late-type spirals (e.g., M81, M101, M33) our infrared observations show that deeply embedded star-forming clusters are extremely rare, at least among the most massive clusters. Such objects comprise of order a percent or less of the young clusters in these galaxies, in contrast to what one might expect from the Milky Way. This suggests that the timescale for molecular cloud breakout in these galaxies is very short, of order 1/100 of the OB stellar lifetimes. By contrast, the Sb spiral NGC 7331, which possess a dense massive molecular ring much like that in the Milky Way, contains roughly a third of all its star formation in deeply embedded clusters in the ring. We are in the process of quantifying these trends for a larger sample of SINGS galaxies (Prescott et $a l$., in preparation), and it is too early to tell what the controlling variables are, though we suspect they are connected to variations in local gas density and interstellar pressure (cf. Dopita et al. 2005).

\section{Concluding Remarks}

When I was invited to give the introductory review for this meeting I was at the same time flattered and intimidated, because my knowledge of the subject of this conference is distinctly from the distance of an extragalactic perspective. But I hope I have conveyed the broad relevance of the issues that will be discussed this week for virtually every facet of Galactic and extragalactic astrophysics, and at the same time provided some sobering reminders of the challenges ahead in understanding how massive star formation proceeds and is controlled over this vast range of physical conditions and galactic environments. As the title of this conference suggests massive star formation has firmly placed itself at the crossroads of astrophysics, and we too have reached a crossroads in our ability to attack this fundamental problem. With the flood of new observations coming in this road ahead promises to be an exciting and productive one.

\section{Acknowledgements}

I am pleased to acknowledge the support of NSF grant AST-0307386 and NASA grant NAG5-8426. Support for this work, part of the Spitzer Space Telescope Legacy Science 
Program, was also provided by NASA through Contract Number 1224769 issued by JPL, Caltech, under NASA contract 1407.

\section{References}

Abel, T., Bryan, G.L., \& Norman, M.L. 2002, Science 295, 93

Bresolin, F., \& Kennicutt, R.C. 1997, AJ 113, 975

Di Matteo, T., Springel, V., \& Hernquist, L. 2005, Nature 443, 604

Dopita, M. et al. 2005, ApJ 619, 755

Figer, D.F. et al. 2002, ApJ 581, 258

Genzel, R. et al. 1998, ApJ 498, 579

Hameed, S., \& Devereux, N. 1999, AJ 118, 730

Heckman, T.M. et al. 2001, ApJ 554, 1021

James, P. et al. 2004, AछA 414, 23

Kennicutt, R.C., Edgar, B.K., \& Hodge, P.W. 1989, ApJ 337, 761

Kennicutt, R.C. 1998a, ARA\&A 36, 189

Kennicutt, R.C. 1998b, ApJ 36, 189

Kennicutt, R.C., Bresolin, F., \& Dolphin, A. 2002, in: D. Geisler, E.K. Grebel, and D. Minniti (eds.), Extragalactic Star Clusters, IAU Symp 207 (ASP Conf Ser) 207, 410

Kennicutt, R.C. et al. 2003, PASP 115, 928

Kobulnicky, H.A., \& Johnson, K.E. 1999, ApJ 527, 154

Kormendy, J., \& Kennicutt, R.C. 2004, ARA\&A 42, 603

Larsen, S. 2002, AJ 124, 1393

Martin, C.L. 1999, ApJ 513, 156

Martin, C.L., Kobulnicky, H.A., \& Heckman, T.M. 2002, ApJ 574, 663

Martin, C.L. 2005, ApJ 621, 227

Martini, P. et al. 2003, ApJS 146, 353

McKee, C.F., \& Williams, J.P. 1997, ApJ 476, 144

Rupke, D.S., Veilleux, S., \& Sanders, D.B. 2002, ApJ 570, 588

Somerville, R.S., \& Primack, J.R. 1999, MNRAS 310, 1087

Stolte, A. 2004, AJ 128, 765

Swinbank, A.M. et al. 2004, ApJ 617, 64

Thilker, D.A. et al. 2005, ApJ (Letters), 619, L79 